\title{
Endoscopic Ultrasound Through-the-Needle Biopsy for the Diagnosis of an Abdominal Bronchogenic Cyst
}

\author{
Jessica Cassiani', Stefano Francesco Crinò ${ }^{2}$, Erminia Manfrin ${ }^{3}$, Matteo Rivelli', Armando Gabbrielli ${ }^{2}$, Alfredo Guglielmi ${ }^{1}$ and \\ Corrado Pedrazzani ${ }^{1}$
}

${ }^{1}$ Department of Surgical Sciences, Dentistry, Gynecology and Pediatrics, Unit of General and Hepatobiliary Surgery, ${ }^{2}$ Department of Medicine, Gastroenterology and Digestive Endoscopy Unit, The Pancreas Institute, ${ }^{3}$ Department of Diagnostic and Public Health, University of Verona Hospital Trust, University of Verona, Verona, Italy

A 57-year-old woman with epigastric pain was diagnosed with a 6-cm abdominal cystic lesion of unclear origin on cross-sectional imaging. Endoscopic ultrasound (EUS) demonstrated a unilocular cyst located between the pancreas, gastric wall, and left adrenal gland, with a regular wall filled with dense fluid with multiple hyperechoic floating spots. A 19-G needle was used to puncture the cyst, but no fluid could be aspirated. Therefore, EUS-guided through-the-needle biopsy (EUS-TTNB) was performed. Histological analysis of the retrieved fragments revealed a fibrous wall lined by "respiratory-type" epithelium with ciliated columnar cells, consistent with the diagnosis of a bronchogenic cyst. Laparoscopic excision was performed, and the diagnosis was confirmed based on the findings of the surgical specimen. Abdominal bronchogenic cysts are extremely uncommon, and a definitive diagnosis is commonly obtained after the examination of surgical specimens due to the lack of pathognomonic findings on cross-sectional imaging and poor cellularity on EUS-guided fine-needle aspiration cytology. EUS-TTNB is useful for establishing a preoperative histological diagnosis, thus supporting the decision-making process. Clin Endosc 2021;54:767-770

Key Words: Bronchogenic cyst; Endoscopic ultrasound-guided fine-needle aspiration; Endoscopic ultrasound-guided throughthe-needle biopsy; Laparoscopy; Pancreatic cyst

\section{INTRODUCTION}

Bronchogenic cysts (BCs) are rare foregut congenital malformations that occur due to abnormal budding of the primitive tracheobronchial tube. ${ }^{1}$ These lesions are commonly identified in the mediastinum, posterior to the carina. BCs in other locations, such as the peritoneal and retroperitoneal spaces, are

Received: July 22, 2020 Revised: September 15, 2020

Accepted: October 17, 2020

Correspondence: Stefano Francesco Crinò

Department of Medicine, Gastroenterology and Digestive Endoscopy Unit, University of Verona Hospital Trust, University of Verona, Piazzale L. Scuro 10, Verona 37134, Italy

Tel: +39-045-812-6191, Fax: +39-045-812-8191, E-mail: stefanocrino@hotmail. com

ORCID: https://orcid.org/0000-0003-4560-8741

(c) This is an Open Access article distributed under the terms of the Creative Commons Attribution Non-Commercial License (http://creativecommons.org/ licenses/by-nc/3.0) which permits unrestricted non-commercial use, distribution, and reproduction in any medium, provided the original work is properly cited. exceedingly rare. Preoperative diagnosis of the cyst hystotype is rarely established via endoscopic ultrasound (EUS)-guided fine-needle aspiration, and resection is usually performed due to uncertainty about the origin of the lesion (the pancreas or stomach). Recently, microforceps (Moray ${ }^{\mathrm{TM}}$; US Endoscopy, Mentor, OH, USA) that can pass through a standard 19-G EUS needle to obtain histological specimens from the cyst wall have become available. Here, we present a case of an abdominal peripancreatic-paracardial BC preoperatively diagnosed by EUS-guided through-the-needle biopsy (EUS-TTNB). Moreover, we highlight cross-sectional imaging and intraoperative findings demonstrating the anatomical relationships of BCs with adjacent structures, which may facilitate diagnosis.

\section{CASE REPORT}

A 57-year-old woman was referred to our unit due to epigastric pain and dyspepsia. Her medical history was un- 
remarkable, and routine laboratory tests showed normal results, except an increased serum amylase level (119 U/L). On abdominal ultrasound, a peripancreatic-paracardial lesion was detected. On computed tomography (CT), a hypodense, $6 \times 4$-cm-sized, oval-shaped lesion of uncertain origin was found along the gastric lesser curvature near the esophagogastric junction, extending toward the diaphragmatic pillar, adjacent to the pancreatic body, and close to the left adrenal gland (Fig. 1A). Magnetic resonance imaging (MRI) demonstrated a hyperdense lesion on T2-weighted sequences, consistent with the radiological characteristics of a cystic lesion.
Upper gastrointestinal endoscopy showed normal results. On EUS, the cyst was found between the pancreas, left adrenal gland, posterior gastric wall, and diaphragm pillar, filled with a dense fluid with a "starry sky" aspect due to multiple floating hyperechoic spots with the comet-tail artifact (Fig. 1B). Due to the apparent high-density cystic fluid content, a 19-G needle was used to puncture the cyst; however, no fluid could be aspirated. Therefore, TTNB was performed (Fig. 1C), obtaining two macroscopically visible specimens from the cyst wall. ${ }^{2}$ No adverse events occurred post-procedure. On histological analysis, the specimens were composed of a fibrous-leiomuscular
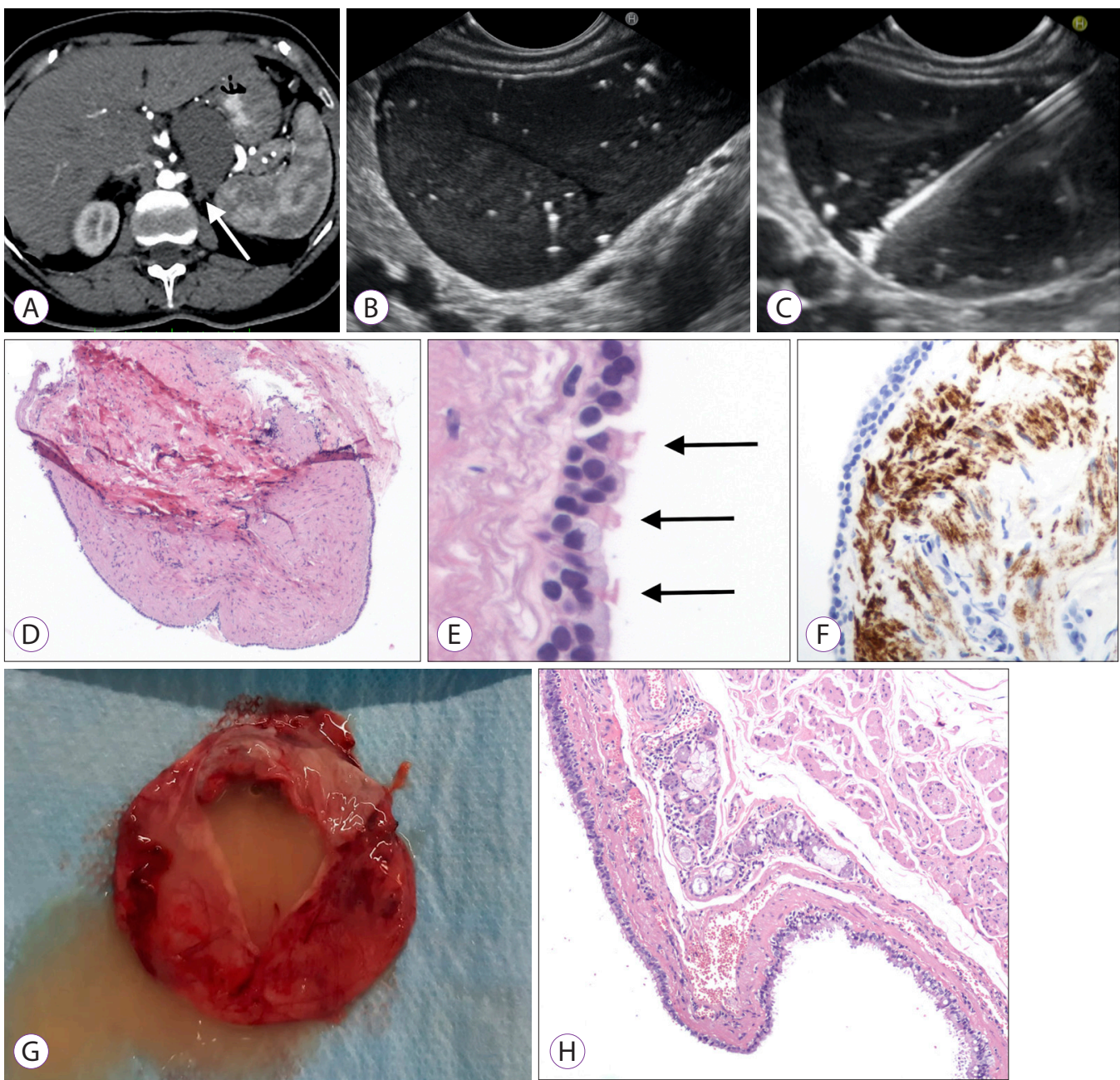

Fig. 1. (A) Computed tomography revealing a well-defined, non-infiltrative lesion of uncertain origin with suspected contact with the diaphragmatic crus (white arrow). (B) Endoscopic ultrasound revealing cystic content with a pseudo solid aspect, with a hypoechoic internal echo pattern with multiple hyperechoic spots and comettail artifacts. (C) Ultrasound view of the Moray ${ }^{\mathrm{TM}}$ (US Endoscopy, Mentor, OH, USA) microforceps during sampling of the cystic wall. (D) A through-the-needle biopsy specimen of the cystic wall composed of cellular stroma supporting a monolayer of the covering epithelium (hematoxilin and eosin [H\&E], $\times 4$ ). (E) The epithelium is composed of cylindrical, ciliated cells (black arrows) and interspersed mucinous cells. Elastic fibers beneath the epithelium and fusiform mesenchymal cells compose the stroma wall (H\&E, $\times 40)$. (F) Immunohistochemical positivity for desmin in leiomuscular cells in the wall (desmin, $\times 40)$. (G) Macroscopic examination of the specimen showing a multilocular cystic lesion filled with a dense mucoid fluid. $(\mathrm{H})$ Histological examination showing a cyst wall of the bronchogenic type, with "respiratory-type" epithelium overlying a muscularis propria layer with accessory mucinous glands (H\&E, $\times 4$ ). 
wall covered by a monolayer of "respiratory-type" epithelium with ciliated columnar cells and interspersed mucinous cells, consistent with the diagnosis of a BC (Fig. 1D, E). The presence of a muscularis propria layer in the cyst wall, highlighted by immunohistochemical positivity for desmin (Fig. 1F), supported the diagnosis.

Laparoscopic excision of the lesion showed close contact between the cyst and the diaphragmatic pillar. Macroscopic examination of the specimen revealed a multilocular cystic lesion filled with a dense mucoid fluid (Fig. 1G). Histology confirmed the preoperative diagnosis of BC (Fig. 1H). The postoperative course was uneventful. The patient was discharged on postoperative day 2 and remained asymptomatic during the 1-year follow-up.

\section{DISCUSSION}

BCs result from abnormal budding of the tracheobronchial analog and the primitive foregut. ${ }^{3}$ Sumiyoshi et al. proposed a theory for the development of subdiaphragmatic BCs. ${ }^{4}$ In early embryologic life, the thoracic and abdominal cavities are linked at the pericardial-peritoneal canal. The canal is later divided by fusion of the pleuroperitoneal membranes (future diaphragm), and a portion of the tracheobronchial tree is pinched off below the diaphragm. ${ }^{4}$

Abdominal BCs are exceedingly rare, and a preoperative diagnosis is seldom obtained due to the lack of pathognomonic characteristics on cross-sectional imaging. CT is unhelpful due to varying density of the lesions. MRI may be helpful because most BCs contain proteinaceous material that characteristically generates high signal intensity on T1-weighted images. ${ }^{5}$ The close contact of a BC with diaphragmatic pillars detected in this case has been previously described and can help physicians to raise the suspect of $\mathrm{BC} .^{5}$

On EUS, we observed an unusual aspect of the cystic content, defined as a "starry sky" aspect due to the presence of multiple hyperechoic spots floating in a dense hypoechoic fluid. This pattern, which is probably correlated with the mucoid content of the cyst, has been previously described in BCs and may help endosonographers suspect a diagnosis of a BC. ${ }^{6,7}$ Cytological diagnosis using EUS-guided fine-needle aspiration specimens is mainly based on the finding of ciliated epithelial cells on fluid cytology. ${ }^{7}$ However, the diagnostic yield of cytology is limited due to the low number of cells dispersed in the cystic fluid. Moreover, as in this case, the high viscosity of the mucoid cystic content does not allow its aspiration. These limitations may be overcome by the current availability

of microforceps that can pass "through-the-needle" to enable a cyst wall biopsy, successfully obtaining specimens suitable for histological evaluation, allowing a more accurate diagnosis than that with cystic fluid cytology. ${ }^{8}$ A recent meta-analysis of patients who underwent TTNB for the diagnosis of pancreatic cysts showed that a diagnosis of the cyst histotype is achieved in approximately two-thirds of cases, ${ }^{9}$ thus, in most cases, the histological diagnosis is available for guiding the decision-making process. ${ }^{10}$ Moreover, compared with fine-needle aspiration cytology, TTNB is feasible even when the viscosity of the cyst content makes fluid aspiration impossible, as described in this study.

With regard to the indication for surgery, some concerns exist regarding recommending excision for asymptomatic BCs, especially if a preoperative diagnosis is obtained. However, some authors recommend surgical excision even in the absence of symptoms to address the risk of infection or malignant degeneration. ${ }^{11}$ Although a majority of abdominal BCs reported to date have been treated with open surgery, a minimally invasive approach seems feasible and safe, supporting the indication of surgical excision in low-risk patients.

In conclusion, the preoperative diagnosis of abdominal BCs remains difficult. The close contact with diaphragmatic pillars documented on cross-sectional imaging as well as the "starry sky" aspect on EUS could represent important diagnostic features of BCs. In this case, we found that EUS-TTNB can be used to obtain specimens for confirming the diagnosis preoperatively, thus supporting the decision-making process.

Conflicts of Interest

The authors have no potential conflicts of interest.

Funding

None.

\section{Author Contributions}

Conceptualization: Stefano Francesco Crinò, Corrado Pedrazzani Supervision: Armando Gabbrielli, Alfredo Guglielmi

Writing-original draft: Jessica Cassiani, SFC, Erminia Manfrin, Matteo Rivelli, CP

Writing-review\&editing: SFC, EM, CP

ORCID

Jessica Cassiani: Stefano Francesco Crinò: Erminia Manfrin: Matteo Rivelli: Armando Gabbrielli: Alfredo Guglielmi: Corrado Pedrazzani:
https://orcid.org/0000-0002-8502-572X https://orcid.org/0000-0003-4560-8741 https://orcid.org/0000-0002-5706-0018 https://orcid.org/0000-0001-9999-0152 https://orcid.org/0000-0001-5875-7952 https://orcid.org/0000-0003-1713-4307 https://orcid.org/0000-0003-2431-6117 


\section{REFERENCES}

1. Barnes NA, Pilling DW. Bronchopulmonary foregut malformations: embryology, radiology and quandary. Eur Radiol 2003;13:2659-2673.

2. Crinò SF, Bernardoni L, Brozzi L, et al. Association between macroscopically visible tissue samples and diagnostic accuracy of EUS-guided through-the-needle microforceps biopsy sampling of pancreatic cystic lesions. Gastrointest Endosc 2019;90:933-943.

3. Gerle RD, Jaretzki A 3rd, Ashley CA, Berne AS. Congenital bronchopulmonary-foregut malformation. Pulmonary sequestration communicating with the gastrointestinal tract. N Engl J Med 1968;278:1413-1419.

4. Sumiyoshi K, Shimizu S, Enjoji M, Iwashita A, Kawakami K. Bronchogenic cyst in the abdomen. Virchows Arch A Pathol Anat Histopathol 1985;408:93-98.

5. Herek D, Erbiş H, Kocyigit A, Yagci AB. Retroperitoneal bronchogenic cyst originating from diaphragmatic crura. Indian J Surg 2015;77(Suppl 3):1397-1398.

6. Klamt A, Di Loreto A, Valle RD, Lukashok HP, Robles-Medranda C. Role of endoscopic ultrasonography in intramural bronchogenic cysts: case reports and review of the literature. Endosc Ultrasound 2012;1:162164.

7. Sato M, Irisawa A, Bhutani MS, et al. Gastric bronchogenic cyst diagnosed by endosonographically guided fine-needle aspiration biopsy. J Clin Ultrasound 2008;36:237-239.

8. Larghi A, Manfrin E, Fabbri C, et al. Interobserver agreement among expert pathologists on through-the-needle microforceps biopsy samples for evaluation of pancreatic cystic lesions. Gastrointest Endosc 2019;90:784-792.e4.

9. Tacelli M, Celsa C, Magro B, et al. Diagnostic performance of endoscopic ultrasound through-the-needle microforceps biopsy of pancreatic cystic lesions: systematic review with meta-analysis. Dig Endosc 2020;32:1018-1030.

10. Crinò SF, Bernardoni L, Gabbrielli A, et al. Beyond pancreatic cyst epithelium: evidence of ovarian-like stroma in EUS-guided throughthe-needle micro-forceps biopsy specimens. Am J Gastroenterol 2018;113:1059-1060.

11. Díaz Nieto R, Naranjo Torres A, Gómez Alvarez M, et al. Intraabdominal bronchogenic cyst. J Gastrointest Surg 2010;14:756-758. 